\title{
Oral leukoplakia manifests differently in smokers and non-smokers
}

\section{Joelma Sousa Lima ${ }^{(a)}$ Décio dos Santos Pinto Jr ${ }^{(a)}$ Suzana Orsini Machado de Sousa(a) \\ Luciana Corrêa(b)}

(a) Oral Pathology Department, School of Dentistry, Univ of São Paulo, São Paulo, SP, Brazil.

(b) General Pathology Department, School of Dentistry, Univ of São Paulo, São Paulo, SP, Brazil.
Declaration of Interests: The authors certify that they have no commercial or associative interest that represents a conflict of interest in connection with the manuscript.

Corresponding Author:

Joelma Sousa Lima

E-mail:joelma.lima@usp.br

Submitted: May 17, 2012

Accepted for publication: Jul 19, 2012

Last revision: Aug 07, 2012

\begin{abstract}
Oral leukoplakias (OL) are potentially malignant lesions that are typically white in color. Smoking is considered a risk factor for developing OL, and dysplastic lesions are more prone to malignant transformation. The aim of this study was to describe the clinical features observed in dysplastic and non-dysplastic OL in both smokers and nonsmokers. A total of 315 cases of OL were retrieved and separated into either dysplastic or non-dysplastic lesions, and these cases were further categorized as originating in either smokers or non-smokers. Frequencies of the type of OL lesion, with respect to whether the patients smoked, were established. The results demonstrated that 131 cases of OL were dysplastic (74 smokers and 57 non-smokers), and 184 were non-dysplastic (96 smokers and 88 non-smokers). For OL cases in smokers for which information about alcohol consumption was also available (84 cases), the results revealed no significant difference in the amount of dysplastic and non-dysplastic lesions. Dysplastic lesions were more frequent in male smokers and in non-smoking females. The median age of smokers with cases of OL was significantly lower than in non-smokers; the lowest median ages were observed for female smokers with dysplastic OL. The most frequent anatomical sites of dysplastic lesions were the floor of the mouth in smokers and the tongue in non-smokers. Dysplastic lesions in smokers were significantly smaller than non-dysplastic lesions in non-smokers. Being a male smoker, being female, being younger, and having smaller lesions were associated with dysplastic features in OL. These clinical data may be important for predicting OL malignant transformation.
\end{abstract}

Descriptors: Leukoplakia, Oral; Risk Factors; Tobacco.

\section{Introduction}

Oral leukoplakia $(\mathrm{OL})$ is a clinical entity defined by the Word Health Organization (WHO) as "a white plaque of questionable risk having excluded (other) known diseases or disorders that carry no increased risk for cancer." ${ }^{\prime 1}$ This type of lesion affects $0.2 \%$ to $4.9 \%$ of the population. ${ }^{2}$ The etiology of OL is variable, and tobacco has been considered a risk factor in a large number of cases, while alcohol, as an independent risk factor, is still questionable. ${ }^{2-7}$ The concept of malignant transformation of OL is quite controversial. Studies have indicated that there is a $36 \%$ chance of malignant transformation if the lesion exhibits dysplastic features. ${ }^{2,7}$ However, when lesions with dysplastic and non-dysplastic fea- 
tures are analyzed together, the percentage of malignant transformation decreases to $0.13 \%$ to $17.5 \% .{ }^{5,8}$ Recent studies have demonstrated that the inclusion of benign alveolar ridge keratosis as a type of OL causes a deviation in the reported percentage of malignant transformation. However, when benign alveolar ridge keratosis is excluded from the OL group, the frequency of dysplasia transforming into squamous cell carcinoma increases from $18 \%$ to $25 \% .^{9}$ Differences in the rates of malignant transformation are also attributed to variations in the criteria used to define OL, such as differences in ethnic and environmental factors. ${ }^{9}, 10$ Risk factors for malignant transformation of OL include being female, having a long duration of OL, having a lesion size greater than $200 \mathrm{~mm}^{2}$, being a non-smoker, having a lesion located on the tongue or the floor of the mouth, having a lesion of a non-homogeneous type, presenting with dysplasia and developing DNA aneuploidy. ${ }^{8,11}$

The histopathological pattern of OL is variable, ranging from atrophy of the epithelium to hyperplasia, either with or without dysplasia. Epithelial dysplasia, if present, exhibits different degrees of severity. $1,3,8,11$

It has been shown that there are differences regarding clinical data when comparing dysplastic and non-dysplastic lesions, mainly in relation to gender, age, anatomical site, alcohol consumption and smoking habits. ${ }^{11-13}$ Therefore, the aim of this retrospective study was to describe the clinical features observed in dysplastic and non-dysplastic OL lesions in both smokers and non-smokers. The purpose of this study was to detect clinical variables observed in OL that could be associated with smoking habits and/or that might be predictors of dysplastic lesions.

\section{Methodology}

All OL cases diagnosed between 2005 and 2009 were retrieved from the files of the Oral Pathology Service at the University of São Paulo. Research was conducted in accordance with protocol 184/2010, approved by the Ethics Committee at the University of São Paulo. Clinical criteria for OL diagnosis were the same as those adopted by the WHO. ${ }^{1}$ Lesions for which clinical history and anatomical sites were indicative of benign alveolar ridge keratosis were

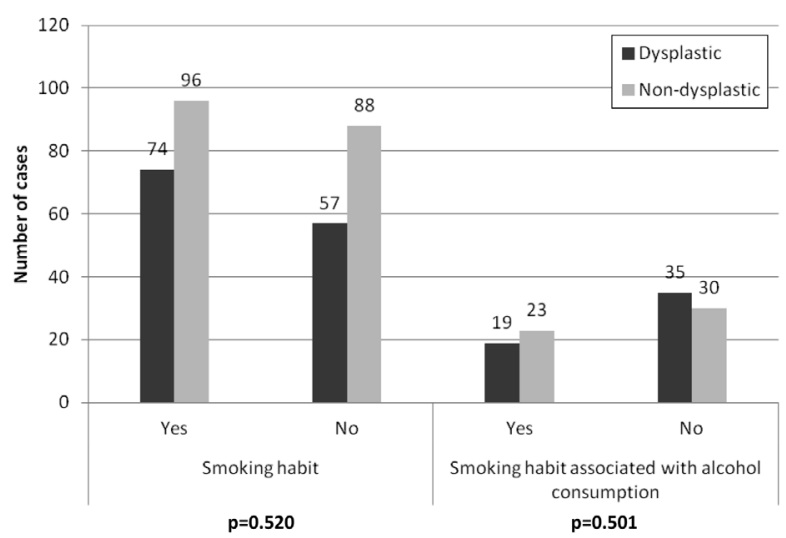

Figure 1 - Frequency of dysplastic and non-dysplastic lesions according to smoking habit and alcohol consumption. (Chi-square test; statistically significant when $p<0.05$.)

excluded. Patients included in the study as smokers were those who had been smoking for at least one year and were presently still smoking. Non-smokers were patients who reported that they had never smoked. ${ }^{4}$ Patients were considered "alcohol consumers" if they presented this chronic habit by the time of the biopsy, and "non-alcohol consumers" if they reported that they never had an alcohol habit. Additionally, based on the histopathological features of the lesions, cases were divided into "dysplastic" (without distinction as to the severity of each dysplasia) and "non-dysplastic" (Figures 1 and 2). ${ }^{6}$ This method of subdivision was adopted because the risk of malignant transformation is higher for dysplastic lesions ${ }^{8,11}$ and because there can be differences in the clinical data when comparing dysplastic and nondysplastic lesions. ${ }^{12}$ Any OL that was histologically diagnosed as a specific disease was not considered in this study.

The data obtained in this study included gender, age, and ethnicity of the patients. For each lesion, the diameter (mm), the duration (months), and the anatomical site were also included. The designation "White" refers to patients of Caucasian origin and their descendants, mainly Europeans; "Black" signifies African people and their descendants; and "Yellow" includes Japanese, Korean, and Chinese people and their descendants. "Other ethnicity" refers to patients presenting a mixture of races. Lesions that had been present for 30 days or less were grouped as having been present for "one month." With regard 


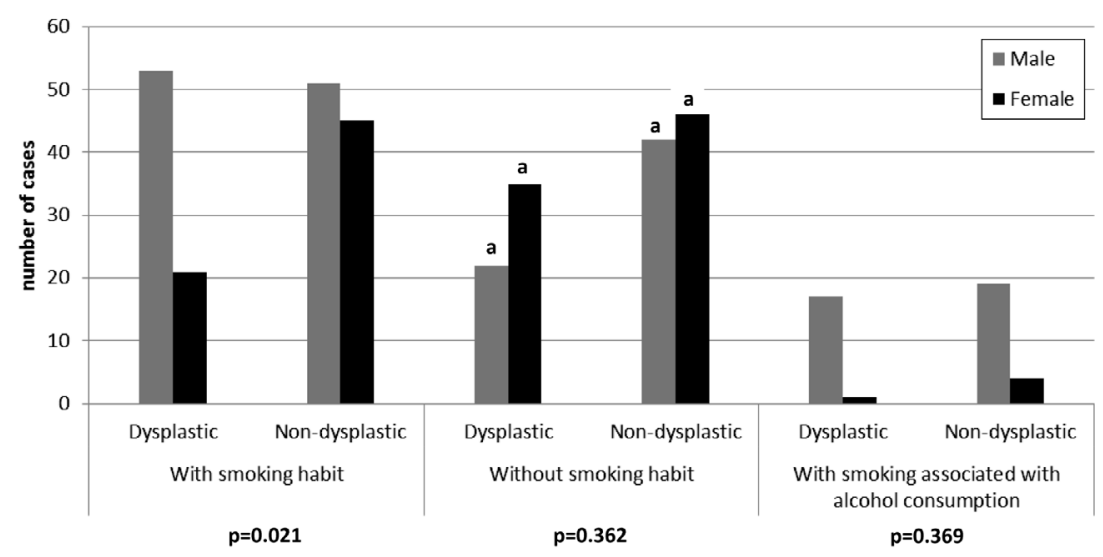

Figure 2 - Frequency of dysplastic and non-dysplastic lesions according to smoking habit, alcohol consumption and gender. (Chi-Square test; statistically significant when $p<0.05$; $a=$ statistically significant when compared with smoking habit/dysplastic lesions.) to anatomical site, the term "tongue" was adopted for both the lateral border and the other tongue regions. The term "palate" includes both the soft and hard palates.

\section{Statistical analysis}

The frequencies of each variable were evaluated with descriptive statistics. The association between the variables in each group was analyzed using the Chi-square test. For numerical data, the Mann-Whitney U-test was applied. Estimation of the smokers-versus-nonsmokers odds ratio was performed for males and for the most frequent anatomical site. The calculations were performed using BioEstat 3.0 (developed by Manuel Ayres, Belém, Brazil) and Microsoft Excel ${ }^{\circledR}$ (Microsoft Corporation, Santa Rosa, USA). Data were considered significant when $\mathrm{p}<0.05$.

\section{Results}

A total of 315 cases of OL were retrieved from the Oral Pathology Service at the University of São Paulo. A total of 131 cases involved dysplastic lesions (74 smokers and 57 non-smokers), and 184 cases described non-dysplastic lesions (96 smokers and 88 non-smokers). The frequencies of lesions, according to the grade of dysplasia, were similar in both groups. The Chi-square test indicated that there was no association between the frequency of dysplastic/non-dysplastic lesions and smoking habits $(\mathrm{p}=0.520)$ or alcohol consumption $(\mathrm{p}=0.501)$ (Figure 1). However, for some variables, such as gender, age, anatomical site of the lesion, and lesion size, some significant associations were found between the smoking habits of the patient and the presence or absence of dysplasia (Figure 2).

Smokers presented a significantly higher frequency of dysplastic lesions in males than in females $(\mathrm{p}=0.021)$. Among non-smokers, females showed a significantly higher frequency of dysplastic lesions than men $(\mathrm{p}<0.01)$. Considering only non-dysplastic lesions, there was no significant association between a smoking habit and gender $(\mathrm{p}=0.559)$. When alcohol consumption was considered, there were also no statistically significant differences between dysplastic and non-dysplastic lesions in women and men (Figure 2). The odds ratio for gender and smoking indicates that male smokers have a four-times greater chance of developing dysplastic lesions than do male non-smokers (Table 1).

The median age in the group of smokers was 55 years for non-dysplastic lesions and 56.5 years for dysplastic lesions. In the group of non-smokers, the median age was 63 years for both dysplastic and nondysplastic lesions. The median age in smokers was significantly lower than in non-smokers (dysplastic lesions, $\mathrm{p}=0.025$; non-dysplastic lesions, $\mathrm{p}=0.004$ ). Considering the group of smokers with dysplastic lesions, women showed a significantly lower median age in comparison with men $(\mathrm{p}=0.032)$. In contrast, women in the group of non-smokers presented a significantly higher median age in comparison with men for both dysplastic $(\mathrm{p}=0.001)$ and non-dysplastic lesions $(\mathrm{p}=0.010)$ (Table 2$)$.

Table 3 shows the frequencies of dysplastic and non-dysplastic lesions along with the smoking hab- 
Table 1- Smoker versus nonsmoker odds ratio for males and the most frequent anatomical sites for dysplastic and nondysplastic lesions.

\begin{tabular}{c|c|c|c|c|c|c}
\hline \multirow{2}{*}{} & \multicolumn{3}{|c|}{ Dysplastic } & \multicolumn{3}{c}{ Non-dysplastic } \\
\cline { 2 - 7 } & OR & Cl & p value & OR & Cl & p value \\
\hline Male & 4.29 & $2.02-9.07$ & $<0.001$ & 1.09 & $0.57-2.10$ & 0.910 \\
\hline Floor of mouth & 2.55 & $0.99-6.55$ & 0.070 & 0.32 & $0.06-1.66$ & 0.292 \\
\hline Tongue & 0.43 & $0.19-0.99$ & 0.070 & 0.36 & $0.15-0.85$ & 0.030 \\
\hline Gingiva & 0.38 & $0.13-1.12$ & 0.128 & 1.36 & $0.72-2.57$ & 0.426 \\
\hline Buccal mucosa & 2.34 & $0.91-6.06$ & 0.118 & 1.83 & $0.90-3.72$ & 0.128 \\
\hline
\end{tabular}

$\mathrm{OR}$ - odds ratio. $\mathrm{Cl}$ - confidence interval. Statistically significant when $\mathrm{p}<0.05$

Table 2 - Median age (minimum and maximum values) of smoking and non-smoking patients with dysplastic and non-dysplastic lesions in general, and in male and female patients.

\begin{tabular}{|c|c|c|c|c|c|c|c|c|c|}
\hline & \multirow{2}{*}{$\begin{array}{l}\text { Smoker } \\
\text { General }\end{array}$} & \multirow{2}{*}{$\begin{array}{c}\text { Non-smoker } \\
\text { General }\end{array}$} & \multirow{2}{*}{$p$ value } & \multicolumn{2}{|c|}{ Smoker } & \multirow{2}{*}{$p$ value } & \multicolumn{2}{|c|}{ Non-smoker } & \multirow{2}{*}{$p$ value } \\
\hline & & & & Male & Female & & Male & Female & \\
\hline Dysplastic & $56.5(28-84)$ & 63 (40-83) & 0.025 & $58(41-84)$ & $53.5(28-83)$ & 0.032 & $50.5(40-83)$ & 67 (42-82) & 0.001 \\
\hline Non-dysplastic & $55 \quad(28-87)$ & 63 (32-89) & 0.004 & 54 (33-87) & $56 \quad(28-79)$ & 0.877 & $57 \quad(32-86)$ & 64 (34-89) & 0.010 \\
\hline
\end{tabular}

Mann-Whitney U-test. Statistically significant when $p<0.05$.

Table 3 - Frequency of dysplastic and non-dysplastic lesions according to smoking habit and anatomical site.

\begin{tabular}{|c|c|c|c|c|c|c|}
\hline & \multicolumn{3}{|c|}{ Smoker } & \multicolumn{3}{|c|}{ Non-smoker } \\
\hline & $\begin{array}{l}\text { Dysplastic } \\
(\%)\end{array}$ & $\begin{array}{c}\text { Non-dysplastic } \\
(\%)\end{array}$ & $p$ value & $\begin{array}{l}\text { Dysplastic } \\
(\%)\end{array}$ & $\begin{array}{c}\text { Non-dysplastic } \\
(\%)\end{array}$ & $p$ value \\
\hline Floor of mouth & $18 \quad(24.3)$ & $2 \quad(2.1)$ & \multirow{8}{*}{$<0.001$} & $8 \quad(14.0)$ & (6.8) & \multirow{8}{*}{0.104} \\
\hline Tongue & $14 \quad(18.9)$ & $9 \quad(9.4)$ & & $22(38.6)$ & $21 \quad(23.9)$ & \\
\hline Gingiva & $6 \quad(8.1)$ & $38 \quad(39.6)$ & & 12 (21.1) & $33 \quad(37.5)$ & \\
\hline Buccal mucosa & $17 \quad(23.0)$ & $27 \quad(28.1)$ & & $8 \quad(14.0)$ & $18 \quad(20.5)$ & \\
\hline Palate & $15 \quad(20.3)$ & $12 \quad(12.5)$ & & $2 \quad(3.5)$ & $(1.1)$ & \\
\hline Multiple sites & $4 \quad(5.4)$ & $3 \quad(3.1)$ & & (5.3) & (4.5) & \\
\hline WI & $\begin{array}{ll}0 & (0.0)\end{array}$ & $5 \quad(5.2)$ & & $2 \quad(3.5)$ & (5.7) & \\
\hline Total & $74(100.0)$ & 96 (100.0) & & $57(100.0)$ & 88 (100.0) & \\
\hline
\end{tabular}

$\mathrm{WI}$ - without information. Chi-square test. Statistically significant when $p<0.05$. it of the patient and the anatomical site of the lesion. Among smokers, the most frequent anatomical sites were the floor of the mouth and the gingiva for dysplastic and non-dysplastic lesions, respectively. Among non-smokers, the most frequent sites were the tongue for dysplastic lesions and the gingiva for non-dysplastic lesions. For smokers, a significant association between anatomical site and dysplastic/ non-dysplastic lesions was observed $(\mathrm{p}<0.001)$. For non-smokers, there was no significant association between lesion frequency and anatomical site $(\mathrm{p}=0.104)$. However, the non-smoking patients had a greater chance to develop OL on the tongue (odds ratio $=0.364$ and $\mathrm{p}=0.03)($ Table 1$)$.

Having a smoking habit correlated with lesions on the floor of the mouth and the buccal mucosa in the cases of dysplasia, but these associations were not statistically significant. Non-dysplastic lesions in smokers were more frequent in the gingiva and buccal mucosa than at other sites, but this was also without significance (Table 3). The frequencies of dysplastic and non-dysplastic lesions with respect to smoking and ethnicity are shown in Figure 3. Whites were most affected in all groups. Due to the low frequency of other races in this study, there was no significant association between smoking habits, 
Figure 3 - Frequency of dysplastic and non-dysplastic lesions according to smoking habit and ethnicity. (Chi-square test; statistically significant when $p<0.05$.)

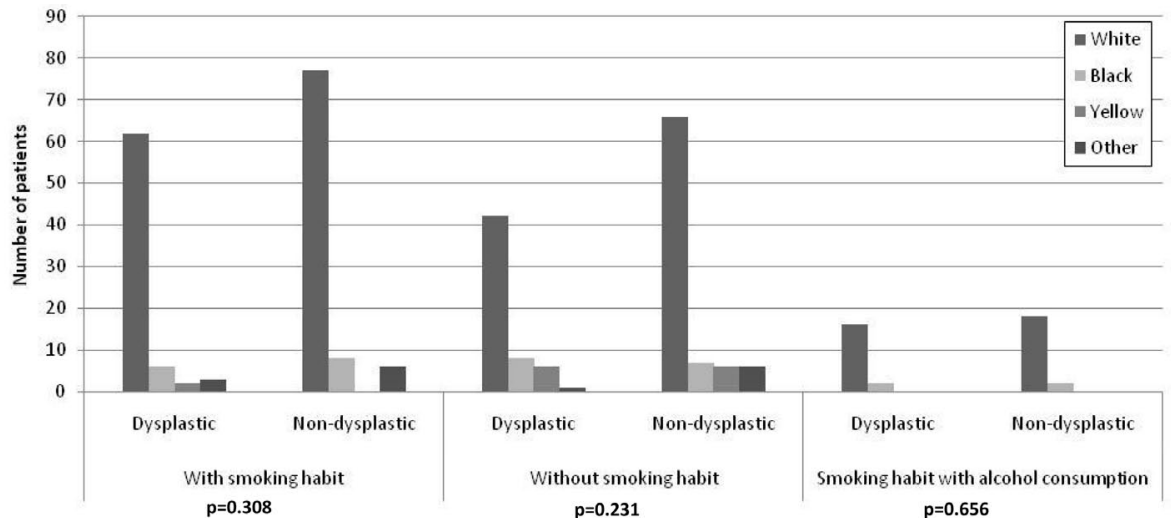

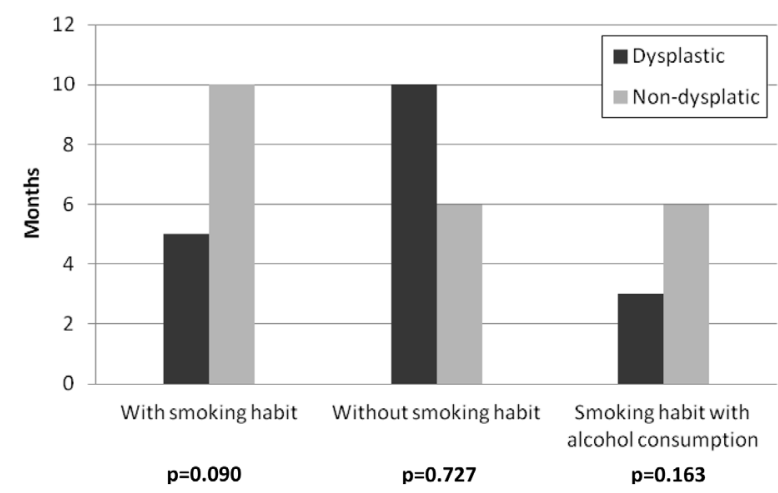

Figure 4 - Median length of lesion duration (in months) from the moment of the clinical diagnosis. (Mann-Whitney U-test; statistically significant when $p<0.05$.)

dysplastic/non-dysplastic lesions, and ethnicity (Figure 3).

The median duration of lesions in smokers was 5 months for dysplastic lesions and 10 months for non-dysplastic lesions (Figure 4). In non-smokers, the median duration was 10 months for dysplastic lesions and 6 months for non-dysplastic lesions. The duration of the lesion had no significant association with smoking habits or histological type (Figure 4), most likely due to the great amount of variability in these data.

The median size of the lesions from patients in this study is shown in Figure 5. For the smoker group, the median diameter of lesions was $10 \mathrm{~mm}$ for both dysplastic and non-dysplastic lesions. In the non-smoker group, the median diameters were $20 \mathrm{~mm}$ for dysplastic and $12.5 \mathrm{~mm}$ for non-dysplastic lesions. There was no significant difference between dysplastic and non-dysplastic lesion size in either smokers or non-smokers (Figure 5). Statisti-

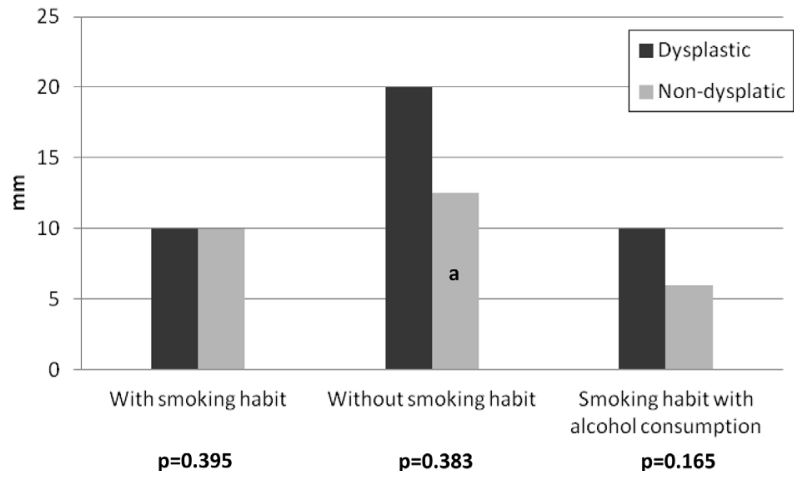

Figure 5 - Lesion diameter ( $\mathrm{mm}$ ) of dysplastic and non-dysplastic lesions according to smoking habit. (Mann-Whitney U-test; statistically significant when $p<0.05$; $a=$ statistically significant when compared with dysplastic lesion in smokers.)

cal significance was observed only when comparing dysplastic lesions in smokers with non-dysplastic lesions in non-smokers; this indicated that the lesions in non-smokers, when non-dysplastic, were significantly larger than the dysplastic lesions in smokers $(\mathrm{p}=0.006)$.

\section{Discussion}

This study focused on clinical data concerning the occurrence of dysplastic and non-dysplastic OL in smokers and non-smokers. Smoking has been correlated with a high frequency of OL in the oral cavity, ${ }^{3,4,7}$ and clinical information about patient gender, the anatomical site of the lesion, the duration of the lesion, and the size of the lesion can be indicative of the potential a lesion has for undergoing malignant transformation. ${ }^{8}$ The most important findings of this study were the associations between dysplas- 
tic lesions and male smokers and between dysplastic lesions and non-smoking women. Additionally, the high frequency of OL on the floor of the mouth, in the gingiva, and in the buccal mucosa of smokers was statistically significant. These results confirmed reports described in the literature indicating that $\mathrm{OL}$ in smokers is associated with male gender and with the floor of the mouth and the buccal mucosa, especially when there is also a habit of alcohol consumption. ${ }^{7}$ However, in the present study, there was no correlation observed between alcohol consumption and dysplasia. Additionally, in non-smokers, OL without dysplasia was likely to develop in women on the tongue. These associations may be important for predicting the risk of dysplastic OL to undergo malignant transformation based only on clinical criteria. This method of risk assessment will prompt pathologists to conduct a careful histopathological diagnosis.

Although the main risk factors for OL are being male and smoking, in this study, we noted a moderate frequency of female non-smokers with dysplastic and non-dysplastic lesions. An increasing frequency of OL has been described in women who do not smoke or consume alcohol,,${ }^{14}$ suggesting the existence of other risk factors for premalignant lesions that show a high potential for malignancy. In the present study, we found no significant association between women, smoking, and dysplastic/ non-dysplastic lesions, except when this information was crossed with the age of the patient. In this case, smoking women with dysplastic lesions had a median age lower than that of smoking men, but non-smoking women had a higher median age than non-smoking men. These findings may indicate that, although not high, the percentage of cases in smoking women could predict the malignant potential of lesions. Therefore, being younger, being female, smoking, and having dysplastic lesions are considered risk factors for malignant transformation of OL lesions. ${ }^{8,11}$

Regarding the patient age of lesion incidence, independent of patient gender, the median age in smokers was lower than in non-smokers for both dysplastic and non-dysplastic lesions. The median ages were between 51 and 60 years and between
61 and 70 years for smokers and non-smokers, respectively. This distribution of ages was similar to that described in other reports. ${ }^{11,14,15}$ However, the Asian population presents a different age distribution for OL. In a Chinese survey that did not record the tobacco habits of patients, OL was considered a disease of the elderly, being present mostly in 60to 69 -year-old patients. ${ }^{10}$ It is difficult to establish an at-risk age for OL development, mainly due to the multiple independent factors that are associated with age and OL, such as chewing betel quid ${ }^{10,15}$ and whether the patient has type 2 diabetes. ${ }^{16,17}$ In our sample, patients 61 years old and above were mainly non-smoking women. As stated above, factors other than smoking may be associated with OL in this group of patients with more advanced age.

The anatomical site of the lesion has also been associated with OL occurrence and the potential for malignant transformation. In the present study, a high frequency of dysplastic lesions was found on the floor of the mouth and in the buccal mucosa for the smoking group and on the tongue for the non-smoking group. Although these sites were also frequently observed in other studies comparing OL clinical data in smokers and non-smokers, ${ }^{11,14,15}$ no odds ratio indicating a strong association between OL and these locations was observed, except for on the tongue in non-smokers. These results were similar to those obtained by Freitas et al. ${ }^{15}$ but different from those of Schepman et al. ${ }^{14}$ who found statistically significant differences in the smoker-versus-non-smoker odds ratio for the floor of the mouth. The association of lesions on the tongue and non-smoking patients has been cited as one of the most common situations that precedes malignant transformation of OL by some authors, but a logistic regression study did not show this association. ${ }^{8}$ A long duration of $\mathrm{OL}^{8}$ and a lesion size $^{3}$ of $>200 \mathrm{~mm}^{2}$ are considered important risk factors for malignant transformation. In the present study, we found no significant association between duration, smoking, and dysplastic/nondysplastic lesions, most likely due to the absence of standardized information and the great variability of the data. Regarding lesion size, the non-dysplastic lesions in non-smokers were significantly larger than the dysplastic lesions in smokers. The significance of 
lesion size and tobacco use in oral epithelial dysplasia is not substantially supported in the literature. Some studies have not established an association between lesion size and clinical aspects and the risk of malignant transformation. ${ }^{7,18}$

The results of this study showed that gender, age, lesion size, and anatomical site correlate with a smoking habit and dysplastic lesions. Men aged between 51 and 60 years of age and lesions on the floor of the mouth and in the buccal mucosa were the most frequent predictors of dysplasia in smokers. In non-smokers, dysplastic lesions were more frequent in women. Although the present study

\section{References}

1. Warnakulasuriya S, Johnson NW, van der Waal I. Nomenclature and classification of potentially malignant disorders of the oral mucosa. J Oral Pathol Med. 2007 Nov;36(10):575-80.

2. Silverman S, Jr., Gorsky M, Lozada F. Oral leukoplakia and malignant transformation. A follow-up study of 257 patients. Cancer. 1984 Feb 1;53(3):563-8.

3. Chiu CT, Li CF, Li JR, Wang J, Chuang CY, Huang SC, et al. Candida invasion and influences in smoking patients with multiple oral leucoplakias-a retrospective study. Mycoses. 2011 Sep;54(5):e377-83.

4. Lee JJ, Hung HC, Cheng SJ, Chen YJ, Chiang CP, Liu BY, et al. Carcinoma and dysplasia in oral leukoplakias in Taiwan: prevalence and risk factors. Oral Surg Oral Med Oral Pathol Oral Radiol Endod. 2006 Apr;101(4):472-80.

5. Cerero-Lapiedra R, Balade-Martinez D, Moreno-Lopez LA, Esparza-Gómez G, Bagán JV. Proliferative verrucous leukoplakia: a proposal for diagnostic criteria. Med Oral Patol Oral Cir Bucal. 2010 Nov 1;15(6):839-45.

6. van der Waal I, Schepman KP, van der Meij EH, Smeele LE. Oral leukoplakia: a clinicopathological review. Oral Oncol. 1997 Sep;33(5):291-301.

7. Schepman KP, van der Meij EH, Smeele LE, van der Waal I. Malignant transformation of oral leukoplakia: a followup study of a hospital-based population of 166 patients with oral leukoplakia from The Netherlands. Oral Oncol. 1998 Jul;34(4):270-5.

8. van der Waal I. Potentially malignant disorders of the oral and oropharyngeal mucosa; present concepts of management. Oral Oncol. 2010 Jun;46(6):423-5.

9. Woo SB, Lin D. Morsicatio mucosae oris--a chronic oral frictional keratosis, not a leukoplakia. J Oral Maxillofac Surg. 2009 Jan;67(1):140-6.

10. Zhang X, Li C, Song Y, Reichart PA. Oral leukoplakia in China: a review. Oral Maxillofac Surg. 2010 Dec;14(4):195202. showed that the localization of lesions on the tongue of non-smokers may indicate the presence of nondysplastic lesions, it is known that white lesions located on the tongue and the floor of mouth are more likely than lesions in the buccal mucosa to undergo malignant transformation.

\section{Conclusion}

The study shows that the anatomical site of OL as well as gender and the presence of epithelial dysplasia should be considered when treating a lesion due to the possibility of a malignant transformation of OL.

11. Jaber MA. Oral epithelial dysplasia in non-users of tobacco and alcohol: an analysis of clinicopathologic characteristics and treatment outcome. J Oral Sci. 2010 Mar;52(1):13-21.

12. Chi AC, Lambert PR 3rd, Pan Y, Li R, Vo DT, Edwards E, et al. Is alveolar ridge keratosis a true leukoplakia? A clinicopathologic comparison of 2,153 lesions. J Am Dent Assoc. 2007 May;138(5):641-51.

13. Hamadah O, Goodson ML, Thomson PJ. Clinicopathological behaviour of multiple oral dysplastic lesions compared with that of single lesions. Br J Oral Maxillofac Surg. 2010 Oct;48(7):503-6.

14. Schepman KP, Bezemer PD, van der Meij EH, Smeele LE, van der Waal I. Tobacco usage in relation to the anatomical site of oral leukoplakia. Oral Dis. 2001 Jan;7(1):25-27.

15. Freitas MD, Blanco-Carrion A, Gandara-Vila P, AntúnezLópez J, García-García A, Gándara Rey JM. Clinicopathologic aspects of oral leukoplakia in smokers and nonsmokers. Oral Surg Oral Med Oral Pathol Oral Radiol Endod. 2006 Aug;102(2):199-203.

16. Lee CH, Ko YC, Huang HL, Chao YY, Tsai CC, Shieh TY, et al. The precancer risk of betel quid chewing, tobacco use and alcohol consumption in oral leukoplakia and oral submucous fibrosis in southern Taiwan. Br J Cancer. 2003 Fev 10;88(3):366-72.

17. Dikshit RP, Ramadas K, Hashibe M, Thomas G, Somanathan T, Sankaranarayanan R. Association between diabetes mellitus and pre-malignant oral diseases: a cross sectional study in Kerala, India. Int J Cancer. 2006 Jan;118(7):453-7.

18. Meisel P, Dau M, Sumnig W, Holtfreter B, Houshmand M, Nauck M, et al. Association between glycemia, serum lipoproteins, and the risk of oral leukoplakia: the populationbased Study of Health in Pomerania (SHIP). Diabetes Care. 2010 Jun;33(6):1230-2. 